\title{
Mudanças em comportamentos relacionados à saúde e indicadores metabólicos em universitários entre 2011 e 2014
}

\author{
Changes in health-related behaviors and metabolic indicators in college students \\ between 2011 and 2014
}

\section{AUTORES \\ Guilherme da Silva Gasparotto ${ }^{1,2}$ (D) \\ Ana Beatriz Pacífico ${ }^{1}$ (iD \\ Edina Maria de Camargo ${ }^{1}(\mathbb{D})$ \\ Wagner de Camposid \\ 1Universidade Federal do Paraná, Setor de Ciências da Educação, Programa de Pós Graduação em Educação, Curitiba, Paraná, Brasil. \\ 2Instituto Federal de Ciência e Tecnologia do Paraná, Curitiba, Paraná, Brasil}

\section{CONTATO}

Guilherme da Silva Gasparotto

guilhermegptt@gmail.com

Rua Governador Jorge Lacerda, 244 casa 4. Curitiba, PR, Brasil. CEP 81050-040.

DOI

10.12820/rbafs.v.22n5p471-78

\section{$(1) \Theta \Theta$ \\ Copyright: This is an open-access article distributed under the terms of the Creative Commons Attribution License ${ }^{\circledR}$, which permits unrestricted use, distribution, and reproduction in any medium, provided that the original author and source are credited.}

\begin{abstract}
RESUMO
Esse estudo teve como objetivo analisar a relação do tempo de exposição ao ambiente universitário com mudanças nos comportamentos relacionados à saúde e indicadores metabólicos em universitários após quatro anos de ingresso no ensino superior. Trata-se de um estudo longitudinal com estudantes de uma Universidade Pública de Curitiba, Paraná, entre 2011 e 2014. A atividade física de lazer foi mensurada por meio do questionário IPAQ versão curta e os comportamentos etilismo e hábitos alimentares foram obtidos por meio do YRBSS. Foram medidos massa corporal, estatura, circunferência de cintura e pressão arterial. Foram avaliados 1197 universitários em 2011 e 455 em 2014. Foi utilizado a regressão linear para verificar a relação do tempo de exposição ao ambiente de graduação com a prática de atividade física, outros comportamentos relacionados à saúde e indicadores metabólicos. O tempo de graduação explicou a variância da prática de AFMV em 16\%, o consumo de bebidas alcoólicas em 13\% e 14\% (consumo em excesso), a medida da cintura em $13 \%$, e a pressão arterial em $9 \%$ (sistólica) e $6 \%$ (diastólica). Os dados demonstram que o tempo de exposição ao ambiente universitário explica parte da variância nos comportamentos relacionados à saúde e indicadores metabólicos em universitários.
\end{abstract}

Palavras-chave: Fatores de risco; Estilo de vida; Estudantes; Comportamentos saudáveis.

\section{ABSTRACT}

This study aims wasto verify the relationship of the exposure time to the university environment with the health-related behaviors and metabolic indicators in college students after four years of entrance in the undergraduate. A longitudinal study was conducted with students from a Public University of Curitiba, Paraná, between 2011 and 2014. The physical activity was measured using the short version IPAQ questionnaire and the behaviors of drinking and eating habits were obtained through of YRBSS. Body mass, height, waist circumference and blood pressure were measured. A total of 1197 college students were evaluated in 2011 and 455 in 2014. Linear regression was used to verify the relationship of the time of exposure to the under graduation environment with the practice of physical activity, and othershealth-related behaviors and metabolic indicators. Undergraduation time explained the AFMV variance in $16 \%$, alcohol consumption in $13 \%$ and $14 \%$ (binge drink), waist measurement in 13\%, and blood pressure in $9 \%$ (systolic) and $6 \%$ (diastolic). This data demonstrate that the time exposure to the university environment explains part of variance in health-related behaviors and metabolic indicators in college students.

Keywords: Risk factors; Life style; Students; Healthy behaviors.

\section{Introdução}

O estilo de vida é objeto de estudo de pesquisadores da área da saúde, por ser um dos mais importantes determinantes da saúde da população ${ }^{1,2}$. Entretanto, apesar de toda informação a respeito do tema, pesquisas comportamentais revelam que entre estudantes universitários parece ocorrer aumento dos índices e da quantidade de hábitos considerados de risco para desenvolvimento de doenças crônico-degenerativas ${ }^{3,4}$.

A identificação de fatores de risco para doenças crô- nicas não transmissíveis poderia contribuir para a prevenção - ou retardo - de algumas doenças em médio e longo prazo $^{3-5}$. A prevalência desse tipo de doenças, não é alta entre jovens adultos ${ }^{6}$, no entanto, a atenção sobre os fatores de risco relacionados a saúde, assim como intervenções para a prevenção, são importantes ${ }^{2}$. A literatura traz diversos estudos que apresentam o perfil do estilo de vida de acadêmicos de diferentes cursos na modalidade presencial, que revelam a presença de fatores de risco entre jovens universitários ${ }^{3,4-7}$. No en- 
tanto, esses estudos são provenientes de delineamento transversal, ou seja, foram coletados em um determinado momento da vida universitária, sem a possível comparação do antes e depois no período de graduação.

Contudo a apresentação de dados por meio de estudos longitudinais, que representem a diferença nos comportamentos relacionados à saúde e indicadores metabólicos em universitários durante o processo de formação (entrada e final da graduação) mostra-se mais adequado, quando comparados ao delineamento transversal, para sugerir alterações desses indicadores ${ }^{8}$. Nesse sentido, o presente estudo apresenta-se relevante, diante do fato de que o ingresso no meio acadêmico gera diferentes transformações, como modificação na carga horária de estudo e rotina de atividades diárias, que podem impactar em aspectos relacionados a saúde dos universitários, mudanças essas que podem perdurar pelo decorrer de suas vidas ${ }^{1,2}$, sendo importante investigar os comportamentos relacionados à saúde e indicadores metabólicos ao longo da formação acadêmi$\mathrm{ca}^{3,4-7}$. Identificadas essas possíveis alterações, durante o período de graduação, é possível sugerir estratégias para intervenções que possibilitem a diminuição desses comportamentos e indicadores. Com base no exposto, o objetivo desse estudo foi verificar a relação do tempo de exposição ao ambiente universitário com os comportamentos relacionados à saúde e indicadores metabólicos em universitários após quatro anos de ingresso no ensino superior.

\section{Métodos}

\section{Delineamento, população e amostra}

Realizou-se um estudo longitudinal descritivo, caracterizado pela observação de uma mesma amostra, em dois pontos da linha do tempo, no primeiro semestre de 2011 e no segundo de $2014^{9}$. A população em estudo consistiu de 5.059 estudantes ingressantes na Universidade Federal do Paraná, Brasil, no ano de 2011. O cálculo da amostra inicial levou em consideração parâmetros de um estudo prévio ${ }^{10}$. Para tal, se utilizou a calculadora do software Epiinfo levando-se em consideração: nível de confiança de $95 \%$; poder amostral de $80 \%$; prevalência de formandos insuficientemente ativos de $60 \%$ e prevalência de ingressantes insuficientemente ativos de $50 \%$.

O processo de seleção por conglomerados se deu em dois níveis e os universitários foram estratificados por número de matriculados em diferentes áreas de conhecimento (Biológicas, Exatas e Humanas) e es- tudantes do diurno (manhã e/ou tarde) e noturno. A amostra final foi dividida proporcionalmente de acordo com esses critérios e as turmas sorteadas aleatoriamente, de acordo com área e turno de estudo. Devido ao desenho do estudo, foi adotada correção por efeito de delineamento (deff) de 1,5 mais $10 \%$ para possíveis perdas de dados, totalizando o valor necessário final de 907 universitários.

Ao final do processo de formação, a perda amostral, comumente apresentada neste tipo de delineamento de estudo, ocorreu por alguns fatores: a) foi impossível avaliar 351 universitários (46,0\%), devido ao não atendimento da coordenação do curso após três contatos via e-mail, telefone e uma visita pessoalmente; b) desistiram de cursar a graduação 283 participantes (38,0\%); c) $108(16,0 \%)$ se recusaram a participar ou não estiveram presentes nos dias de coleta. Com isso, a amostra final correspondeu a aproximadamente $40 \%$ da amostra. O poder da amostra final foi calculado a posteriori utilizando o software GPower 3.1.1, que permitiu verificar um poder de 99\%, com valor alpha de 5\%, para identificar coeficientes de regressão significativos para valores de $b e t a=0,06$ (variável PAD menor resultado significativo encontrado nesse estudo).

\section{Instrumentos e Variáveis}

A variável independente tempo de exposição no ambiente de graduação foi utilizada de forma contínua em meses na graduação após matrícula de ingresso na universidade. A classe econômica dos alunos foi realizada por meio da metodologia da ABEP, Associação Brasileira de Empresas de Pesquisa. Segundo a metodologia, os indivíduos foram distribuídos em classes A, B, C, D e E.

Entre as variáveis dependentes, para acompanhamento do nível de atividade física foi utilizado o IPAQ (International Physical Activity Questionnaire) versão curta, validado internacionalmente para população jovem adulta e traduzido para a língua portuguesa ${ }^{11}$. O tempo de atividade física de lazer, moderada vigorosa (AFMV) e atividade física vigorosa (AFV) foi considerado em minutos por semana.

Para obtenção dos comportamentos etilismo e hábitos alimentares, foram utilizadas as questões específicas desses comportamentos, contidas no Youth Risk Behavior Surveillance System (YRBSS), desenvolvido pelo Centers for Disease Control and Prevention (CDC), previamente validado para universitários brasileiros e utilizado em outros estudos com população similar ${ }^{12,13}$. 
Para o consumo de bebidas alcoólicas, foram questionados se, nos últimos trinta dias, foi ingerida pelo menos uma dose, e, nesse período, se foram consumidas cinco ou mais doses numa mesma ocasião. No caso de resposta positiva, em quantos dos últimos trinta dias houve consumo. Sendo assim, as variáveis consumo de bebidas alcoólicas e consumo em excesso foram apresentadas de forma contínua em dias. Sobre hábitos alimentares, os universitários foram questionados se, no dia anterior à pesquisa, houve o consumo de frutas, saladas verdes e/ou vegetais, salgadinhos (chips ou similares) e bolos, tortas ou doces em geral e a quantidade de porções consumidas.

Foram realizadas medidas antropométricas de acordo com o sugerido por Tarnus e Bourdon ${ }^{14}$ : estatura, massa corporal e medida da circunferência da cintura. Para a aferição da estatura foi utilizada uma fita métrica metálica portátil com resolução de $0,1 \mathrm{~cm}$. Foi realizada medida entre os planos que tangenciam o vértex e a planta dos pés, com o avaliado em posição ortostática, descalço, com a menor quantidade de roupa possível. Para determinação da massa corporal, foi utilizada uma balança portátil com resolução de $0,1 \mathrm{Kg}$, seguindo os critérios de posição da estatura. $\mathrm{O}$ índice de massa corporal (IMC) foi definido pela relação equivalente da massa corporal pela estatura ao quadrado, conforme a equação padrão: IMC $=$ massa corporal $(\mathrm{kg}) /$ estatura $\left(\mathrm{m}^{2}\right)$. A circunferência da cintura (CC) foi obtida com uma fita antropométrica metálica $(\mathrm{cm})$, sendo medida no plano horizontal, no ponto médio entre o íleo-cristal e a última costela flutuante. E utilizada de forma continua na análise.

A pressão arterial sistólica (PAS) e a diastólica (PAD) foram verificadas por meio do método auscultatório, segundo recomendação da Sociedade Brasileira de Cardiologia ${ }^{15}$ no braço esquerdo de cada avaliado, mediante utilização de um estetoscópio e de um esfigmomanômetro analógico (marca BD) calibrado com uma semana de antecedência ao início da coleta dos dados. Os estudantes ficaram, por pelo menos cinco minutos, em repouso, sentados com as pernas descruzadas, pés apoiados no chão e dorso recostado na cadeira antes da medição. $\mathrm{O}$ braço foi posicionado na altura do coração com a palma da mão voltada para cima e o cotovelo ligeiramente fletido. $\mathrm{O}$ manguito do esfigmomanômetro foi posicionado cerca de 2 a $3 \mathrm{~cm}$ da fossa cubital, e a campânula do estetoscópio sobre a artéria braquial sem compressão excessiva. Todas as medidas antropométricas e de pressão arterial foram realizadas pelo mesmo avaliador e realizadas uma única vez.

A presente pesquisa foi aprovada pelo Comitê de
Ética em Pesquisa com seres humanos do setor de Ciências da Saúde da Universidade Federal do Paraná, estando de acordo com a resolução n. 466/2012 do Conselho Nacional de Saúde, sob o número de registro CEP/SD: 1043.168.10.11.

\section{Tratamento estatístico}

Os dados foram tabulados e analisados com utilização do Statistical Package for the Social Sciences (SPSS versão 18.0). A normalidade dos dados foi verificada por meio do teste de Kolgomorov-Smirnov. Para comparar as prevalências das variáveis sociodemográficas no início (2011) e final do estudo (2014) foi utilizado teste do qui-quadrado de Mantel-Haenszel ou exato de Fisher, quando necessário. Para análise de averiguação de possíveis alterações nos comportamentos de risco e indicadores metabólicos foram utilizados valores contínuos da média, complementada com o desvio padrão para: tabagismo, consumo de bebidas alcoólicas, hábitos alimentares, IMC, CC e PA, mediante teste $\mathrm{t}$ de Student para medidas repetidas. Também foi utilizado o teste de Wilcoxon pareado para diferenças entre as medianas, com a amplitude interquartil das variáveis AFMV e AFV entre 2011 e 2014.

A regressão linear foi utilizada para verificar a relação do tempo de exposição ao ambiente de graduação com a prática de atividade física e as outras variáveis dependentes. $\mathrm{Na}$ análise da relação do tempo em exposição ao ambiente de graduação (em meses) com AFMV e AFV foi realizado controle das variáveis confundidoras: idade, sexo, nível socioeconômico e IMC. Já, para análise da relação do tempo em exposição ambiente de graduação com os outros fatores de risco foi feito controle das variáveis: idade, sexo, nível socioeconômico. $\mathrm{O}$ nível de significância estipulado para as análises foi de $5 \%$.

\section{Resultados}

Dentre os 5.059 universitários elegíveis para compor a amostra, foram avaliados 1.221 em 2011. Após a exclusão dos questionários respondidos incorretamente ou incompletos, totalizaram 1.197 universitários a amostra inicial, que apresentaram média de idade de 18,3 $( \pm 2,8)$ anos. A segunda etapa de avaliação, ocorreu em 2014, em que, daqueles avaliados inicialmente, 455 foram reavaliados, nessa etapa apresentaram média de idade de 22,1 ( $\pm 3,1$ anos). A descrição e comparação das prevalência de fatores sociodemográficos, no início do estudo (2011) e final (2014), estão expostas na tabela 1.

$\mathrm{Na}$ tabela 2 observam-se os valores das alterações 
Tabela 1 - Descrição e comparação das prevalência de fatores sociodemográficos, no início e final do estudo. Curitiba, 2011 - 2014 ( $\mathrm{n}=455$ ).

\begin{tabular}{|c|c|c|c|c|c|}
\hline \multirow{2}{*}{ Variáveis } & Prevalência (n) & IC 95\% & Prevalência (n) & IC 95\% & \multirow{2}{*}{$\mathrm{p}$} \\
\hline & \multicolumn{2}{|c|}{$2011(n=1197)$} & \multicolumn{2}{|c|}{$2014(n=455)$} & \\
\hline \multicolumn{6}{|l|}{ Idade (anos) } \\
\hline$<18$ & $18,5(222)$ & $16,4-20,7$ & & & $0,01^{*}$ \\
\hline$\geq 18$ & 81,5 (975) & $79,3-83,6$ & $100(00)$ & & \\
\hline \multicolumn{6}{|l|}{ Sexo } \\
\hline Feminino & $50,0(599)$ & $47,3-53$ & $56(255)$ & $52,2-59,4$ & $0,03^{*}$ \\
\hline Masculino & $50,0(598)$ & $47-52,7$ & $44(200)$ & $40,8-48,3$ & \\
\hline \multicolumn{6}{|l|}{ Estado civil } \\
\hline Solteiro & $92,9(1112)$ & $91,4-94,3$ & $89,8(406)$ & $83-94,1$ & 0,38 \\
\hline Casado & $7,1(85)$ & $5,7-8,6$ & $10,2(49)$ & $7,2-14,6$ & \\
\hline \multicolumn{6}{|c|}{ Área de estudo } \\
\hline Humanas & $38(455)$ & $35,3-40,9$ & $30,3(138)$ & $26,1-34,8$ & \multirow{3}{*}{$0,04^{*}$} \\
\hline Exatas & $37,3(446)$ & $34,6-40,1$ & $44,1(138)$ & $41,3-49,9$ & \\
\hline Biológicas & 24,7 (296) & $22,1-27,2$ & $25,6(130)$ & $20,2-28,8$ & \\
\hline \multicolumn{6}{|c|}{ Classe econômica } \\
\hline$A$ e $B$ & $75,1(899)$ & $72,7-77,5$ & $69,1(314)$ & $66,4-72,0$ & \multirow{3}{*}{0,69} \\
\hline $\mathrm{C}$ & $22,7(272)$ & $20,4-25,1$ & $27,7(126)$ & $22,4-31,8$ & \\
\hline $\mathrm{D}$ e $\mathrm{E}$ & $2,2(26)$ & $1,3-3,1$ & $3,2(05)$ & $0,3-6,9$ & \\
\hline \multicolumn{6}{|c|}{ Período de estudo } \\
\hline Manhã & $28,5(341)$ & $25,8-31,1$ & $35,6(162)$ & $28,4-40,0$ & \multirow{4}{*}{0,05} \\
\hline Tarde & $7,6(91)$ & $6,1-9,1$ & $3,3(15)$ & $0,3-7,1$ & \\
\hline Noite & $25,5(303)$ & $23,1-27,8$ & 20,5 (93) & $14,9-27,2$ & \\
\hline Integral & $38,6(462)$ & $35,9-41,3$ & $40,6(185)$ & $35,3-46,1$ & \\
\hline
\end{tabular}

IC: Intervalo de Confiança; ${ }^{*} \mathrm{p}<0,05$.

nas medianas do tempo de prática de AFMV em ambos os sexos e de AFV entre as mulheres. A circunferência de cintura, a pressão arterial sistólica e o consumo de doces aumentaram em ambos os sexos. $\mathrm{O}$ consumo de vegetais cozidos diminuiu, também para ambos os sexos. Já o aumento do consumo de bebidas alcoólicas e seu uso excessivo ocorreu somente no sexo masculino.

O modelo de regressão linear contendo o tempo de graduação mais as variáveis de ajuste explicou $35 \%$ da variância do tempo em AFMV e 24\% da variância do tempo em AFV. Entretanto, como exposto na tabela 3, o tempo de graduação explicou somente a prática de AFMV.

Para os comportamentos relacionados à saúde e indicadores metabólicos, o modelo contendo o tempo de graduação e as variáveis de ajuste explicou 32,0\% do consumo de bebidas alcoólicas, $36,0 \%$ do consumo dessas bebidas em excesso, 28,0\% da variância da CC, $17 \%$ da variância da PAS e $12,0 \%$ da PAD. Os outros indicadores não apresentaram relação com o tempo de exposição ao ambiente de graduação. A contribuição do tempo de graduação na explicação das variâncias dos comportamentos relacionados à saúde e indicadores metabólicos está exposta na tabela 4.

\section{Discussão}

As medidas realizadas no início da graduação e próximo do fim do curso identificaram mudanças nos comportamentos relacionados à saúde e nos indicadores metabólicos dos universitários. Sobre a atividade física, o modelo contendo o tempo de exposição ao ambiente de graduação mais as variáveis de ajuste explicou 35,0\% da variância do tempo em AFMV e 24,0\% da variância do tempo em AFV. Entretanto, sozinho, o tempo de graduação explicou somente a prática de AFMV com uma variância de $16,0 \%$. Para os comportamentos relacionados à saúde e indicadores metabólicos, o modelo contendo o tempo de graduação e as variáveis de ajuste explicaram 32,0\% do consumo de bebidas alcoólicas, $36,0 \%$ do consumo dessas bebidas em excesso, 28,0\% da variância da CC, $17,0 \%$ da variância da PAS e 12,0\% da PAD. Os outros fatores não apresentaram relação com o tempo de exposição ao ambiente de graduação. Entretanto, sozinho, a contribuição do tempo de exposição, na explicação das variâncias do consumo de bebidas alcoólicas foi de 13,0\% e 14,0\% (consumo em excesso), na medida da cintura foi de $13,0 \%$, e na pressão arterial foi de 9,0\% (sistólica) e 6,0\% (diastólica).

A diminuição no tempo de atividade física corrobo- 
Tabela 2 - Comparação das medianas e amplitude interquartil de AFMV e AFV e das médias e desvio padrão dos comportamentos de risco e indicadores metabólicos, obtidos no início e final do estudo para os sexos masculino e feminino - Curitiba, $2011-2014$ ( $\mathrm{n=} 455$ ).

\begin{tabular}{|c|c|c|c|c|}
\hline \multirow{2}{*}{ Variáveis } & 2011 & 2014 & \multirow{2}{*}{ Variação (Mediana) } & \multirow{2}{*}{$\mathrm{p}$} \\
\hline & Mediana (AI) & Mediana (AI) & & \\
\hline \multicolumn{5}{|l|}{ Feminino } \\
\hline AFMV (minutos/semana) & $120(300)$ & $100(310)$ & -20 & $<0,01^{*}$ \\
\hline AFV (minutos/semana) & $25(60)$ & $20(55)$ & -05 & $0,02^{*}$ \\
\hline \multicolumn{5}{|l|}{ Masculino } \\
\hline AFMV (minutos/semana) & $150(390)$ & $120(300)$ & -30 & $<0,01^{*}$ \\
\hline \multirow[t]{2}{*}{ AFV (minutos/semana) } & $35(180)$ & $32(160)$ & -03 & 0,88 \\
\hline & Média (DP) & Média (DP) & Variação & $\mathrm{p}$ \\
\hline \multicolumn{5}{|l|}{ Feminino } \\
\hline $\operatorname{IMC}\left(\mathrm{kg} / \mathrm{m}^{2}\right)$ & $22,7(3,9)$ & $23,5(3,8)$ & $+0,8$ & 0,06 \\
\hline $\mathrm{CC}(\mathrm{cm})$ & $70,9(8,1)$ & $75,1(8,8)$ & $+4,2$ & $<0,01^{*}$ \\
\hline PAS (mmHg) & $108(12)$ & $111(12)$ & +03 & $0,03^{*}$ \\
\hline PAD (mmhg) & $72(9)$ & $72(8)$ & - & 0,92 \\
\hline Consumo de frutas (porções) & $1,13(1,14)$ & $1,31(1,07)$ & $+0,18$ & 0,07 \\
\hline Consumo de saladas (porções) & $1,03(0,82)$ & $1,12(0,77)$ & $+0,9$ & 0,13 \\
\hline Consumo de vegetais cozidos (porções) & $0,82(0,67)$ & $0,71(0,72)$ & $-0,11$ & $0,04^{*}$ \\
\hline Consumo de doces (porções) & $0,97(1,12)$ & $1,22(084)$ & $+0,25$ & $0,01^{*}$ \\
\hline Consumo de salgados (porções) & $0,47(0,75)$ & $0,39(0,63)$ & $-0,08$ & 0,14 \\
\hline Consumo de bebidas alcoólicas (dias) & $2,94(4,12)$ & $3,15(4,74)$ & $+0,21$ & 0,51 \\
\hline Consumo de bebidas em excesso (dias) & $0,93(2,55)$ & $1,00(2,38)$ & $+0,07$ & 0,72 \\
\hline \multicolumn{5}{|l|}{ Masculino } \\
\hline $\operatorname{IMC}\left(\mathrm{kg} / \mathrm{m}^{2}\right)$ & $23,6(4,2)$ & $24,3(4,1)$ & $+0,7$ & 0,12 \\
\hline $\mathrm{CC}(\mathrm{cm})$ & $79(9,7)$ & $83(9,6)$ & +04 & $<0,01^{*}$ \\
\hline PAS (mmHg) & $117(12)$ & $121(13)$ & +04 & $0,02^{*}$ \\
\hline PAD (mmhg) & $77(9)$ & $75(9)$ & -02 & 0,15 \\
\hline Consumo de frutas (porções) & $0,97(1,14)$ & $1,16(1,1)$ & $+0,19$ & 0,17 \\
\hline Consumo de saladas (porções) & $1,03(0,8)$ & $0,98(0,7)$ & $-0,05$ & 0,92 \\
\hline Consumo de vegetais cozidos (porções) & $0,62(0,6)$ & $0,51(0,6)$ & $-0,11$ & $0,04^{*}$ \\
\hline Consumo de doces (porções) & $0,82(1,1)$ & $1,08(0,9)$ & $+0,26$ & $0,03^{*}$ \\
\hline Consumo de salgados (porções) & $0,41(0,7)$ & $0,38(0,5)$ & $-0,13$ & 0,53 \\
\hline Consumo de bebidas alcoólicas (dias) & $4,11(5,1)$ & $5,93(5,6)$ & $+1,82$ & $<0,01^{*}$ \\
\hline Consumo de bebidas em excesso (dias) & $1,62(2,9)$ & $2,34(3,4)$ & $+0,72$ & $0,02^{*}$ \\
\hline
\end{tabular}

DP: Desvio padrão; AI: Amplitude Interquartil; IMC: Índice de Massa Corporal; $\mathrm{Kg} / \mathrm{m}^{2}$ : Quilograma por metro ao quadrado; CC: Circunferência de cintura; cm: centímetros; PAS: Pressão arterial sistólica; PAD: Pressão arterial diastólica; mmHg: Milímetros de mercúrio. *p<0,05.

Tabela 3 - Relação do tempo de exposição dos estudantes ao ambiente da graduação com o tempo em AFMV e AFV ajustados por sexo, idade, nível socioeconômico e IMC - Curitiba, $2011-2014(\mathrm{n}=455)$.

\begin{tabular}{llc}
\hline Variáveis & Beta & IC 95\% \\
\hline AFMV (min./semana) & 0,16 & $0,11-0,21$ \\
AFV (min./semana) & $0,04 * 01^{*}$ & $-0,94-0,12$ \\
\hline
\end{tabular}

AFMV: Atividade Física Moderada-Vigorosa; AFV: Atividade Física Vigorosa; Intervalo de Confiança. *p $<0,05$.

Tabela 4 - Relação do tempo de exposição dos estudantes ao ambiente da graduação com fatores relacionados à saúde ajustados por sexo, idade, nível socioeconômico - Curitiba, $2011-2014(\mathrm{n}=455)$.

\begin{tabular}{|c|c|c|c|}
\hline Variáveis & Beta & IC 95\% & $\mathrm{P}$ \\
\hline $\operatorname{IMC}\left(\mathrm{kg} / \mathrm{m}^{2}\right)$ & $-0,09$ & $-0,15-0,11$ & 0,72 \\
\hline $\mathrm{CC}(\mathrm{cm})$ & 0,13 & $0,08-0,17$ & $0,01^{*}$ \\
\hline PAS (mmHg) & 0,09 & $0,04-0,14$ & $0,01^{*}$ \\
\hline PAD (mmhg) & 0,06 & $0,01-0,10$ & $0,03^{*}$ \\
\hline Consumo de bebidas alcoólicas (dias) & 0,13 & $0,09-0,18$ & $0,01^{*}$ \\
\hline Consumo de bebidas em excesso (dias) & 0,14 & $0,10-0,17$ & $0,01^{*}$ \\
\hline Consumo de frutas (porções) & 0,04 & $0,01-0,07$ & 0,14 \\
\hline Consumo de saladas (porções) & 0,08 & $0,03-0,12$ & 0,65 \\
\hline Consumo de vegetais cozidos (porções) & 0,06 & $0,01-0,11$ & 0,12 \\
\hline Consumo de doces (porções) & 0,06 & $0,02-0,09$ & 0,25 \\
\hline Consumo de salgados (porções) & $-0,07$ & $-0,03-0,11$ & 0,52 \\
\hline
\end{tabular}

IC: Intervalo de Confiança; IMC: Índice de Massa Corporal; $\mathrm{kg} / \mathrm{m}^{2}$ : Quilograma por metro ao quadrado; CC: Circunferência de cintura; cm: centímetros; PAS: Pressão arterial sistólica; PAD: Pressão arterial diastólica; mmHg: Milímetros de mercúrio. *p<0,05. 
ra com outros estudos ${ }^{16,17}$, como o de Jacobsen et al. ${ }^{17}$, que acompanhou universitários suíços durante dois anos e verificou diminuição de nove minutos na prática de atividade física diária. Small et al. ${ }^{16}$, acompanharam ingressantes em uma universidade norte americana e, após sete semestres, verificaram a diminuição média diária de oito minutos gastos em atividades físicas. Os resultados demonstram que os níveis de atividade física reduzem com a exposição ao ambiente de graduação ${ }^{16,17}$, porém o presente estudo revelou que essa mudança foi explicada por uma parcela de $16,0 \%$ pela exposição ao tempo na universidade, mostrandoque, grande parte das alterações na prática de atividade física podem estar associadas a outras variáveis explicativas. Já foi demonstrado que algumas características sociodemográficas podem estar relacionadas à prática de atividade física ${ }^{18}$. No presente estudo, quando as variáveis de ajuste (idade, sexo, classe econômica e IMC) foram incluídas, o modelo explicou mais que o dobro da variância, comparado ao tempo em exposição ao ambiente universitário, isoladamente.

O consumo de bebidas alcoólicas em estudantes universitários é uma das variáveis mais estudadas nesta população, visto que, além de problemas relacionados a ocorrência de doenças, este comportamento está associado a diversos outros riscos, como prática sexual não segura e direção imprudente ${ }^{19}$. No presente estudo, houve aumento do consumo entre os homens e o tempo de exposição ao ambiente da graduação explicou a variância em 13,0\% e 14,0\% (consumo excessivo) do consumo de bebidas alcoólicas. Segundo autores norte-americanos ${ }^{20}$, após quatro anos de acompanhamento foi possível observar que tanto o número de doses consumidas em um mesmo evento, quanto o número de eventos que favorecem o consumo, aumentaram ${ }^{20}$. Em outro trabalho, Iwamoto et al. ${ }^{21}$, mostraram que o número de episódios de consumo excessivo de bebidas alcoólicas, em três meses, progrediu de quatro para oito.

Halperin et al. ${ }^{22}$, analisaram os fatores que contribuem para aumento do consumo de bebidas alcoólicas e o excesso ingerido pelos estudantes, bem como a utilização de outras substâncias tóxicas, e identificaram diversos fatores relacionados. Segundo esses autores, fatores determinantes para iniciação ou intensificação destes comportamentos são o histórico familiar, a personalidade do indivíduo, necessidade de socialização com seus pares e a exposição aos ambientes favorecedores da ação ${ }^{22}$. Neste sentido, com passar do tempo, com relação ao ambiente, o estudante tem a possibilidade de contato com maior número de colegas, consequentemente, mais oportunidades e companhias para bares e festas universitárias. Estes fatores podem ser agravados ainda, por exemplo, pela necessidade do jovem morar sozinho ou dividir a casa com outros participantes, o que gera um sentimento de liberdade e independência ${ }^{22}$.

A respeito do $\mathrm{IMC}$ e $\mathrm{CC}$, estudos já reportaram aumento da massa corporal de acadêmicos no decorrer da graduação ${ }^{23}$. Lloyd-Richardson et al. ${ }^{24}$, verificaram que a massa corporal de universitários aumentou em 3,5 quilogramas, após o primeiro ano de curso. Gropper et al. ${ }^{8}$, identificaram elevação do valor médio do IMC em $\mathrm{kg} / \mathrm{m}^{2}$ de 22,9 para 24,4, ao longo dos quatro anos da graduação. Já Lynn ${ }^{23}$ demonstrou aumento do IMC em $\mathrm{kg} / \mathrm{m}^{2}$ de 23 para 27,6 após os mesmos quatro anos, em uma amostra de 2.353 estudantes.

Como já se apresenta bem definido, o excesso de peso corporal é fator de risco importante para o desenvolvimento de doenças cardiovasculares. Entretanto, a disposição da gordura corporal parece ter maior influência no desencadeamento deste tipo de condição ${ }^{25}$. No presente trabalho, a CC teve aumento de aproximadamente quatro centímetros, ao fim do estudo e ainda, o tempo de graduação explicou 13,0\% dessa variação. Morrow et al. ${ }^{26}$ identificaram aumento médio de um centímetro na circunferência da cintura de estudantes, após o primeiro ano de faculdade. Foi possível verificar neste estudo alterações em hábitos alimentares e prática de atividade física em diversos subgrupos, que podem ter contribuído para aumento na medida da circunferência da cintura. Outros autores discutem, há décadas, o aumento do consumo energético, da massa corporal e consequentemente da CC entre universitários ingressantes ${ }^{27}$. $\mathrm{O}$ fenômeno de alterações bruscas nessas variáveis no primeiro ano de curso foi nomeado de Freshman 15, em referência a aproximadamente 15 libras de aumento da massa dos estudantes em relação ao primeiro ano universitário ao decorrer do curso ${ }^{27}$.

Sobre a PAS e PAD, não é comum na literatura verificar altos valores da pressão arterial de estudantes, que possam indicar risco, devido, principalmente, a idade baixa dessa população ${ }^{28,29}$. Entretanto, 9,0\% (PAS) e 6\% (PAD) da variância da pressão arterial foi explicada pelo período de formação. Deve-se considerar o conjunto de alterações que pode ter impactado nesta elevação dos valores pressóricos: aumento do consumo de bebidas alcoólicas, diminuição do consumo de frutas, saladas e/ ou vegetais, aumento de consumo de salgados, aumento dos valores de CC, todas estas variáveis alteradas são retratadas pela literatura como associadas à elevada $\mathrm{PA}^{30}$.

$\mathrm{O}$ presente estudo apresenta limitações que devem 
ser consideradas ao interpretar os resultados. Apesar da amostra ser representativa no primeiro momento da coleta, ao final sofreu importante perda amostral, contudo, o cálculo de poder apontou o valor de 99,0\% para a variável com resultado de menor relação (PAD), o que permitiu identificar importantes alterações noscomportamentos relacionados à saúde e indicadores metabólicos. O emprego de medidas auto relatadas depende da veracidade e poder recordatório das respostas do entrevistado. As avaliações foram realizadas em contexto de aula, o que pode influenciar nos valores de algumas medidas, principalmente a pressão arterial, que pede um protocolo mais rigoroso para medição. $\mathrm{Na}$ tentativa de minimizar esses vieses, os pesquisadores foram treinados para auxiliar os universitários na resposta dos questionários e a medida da PA foi realizada seguindo orientação de posição e repouso sentado, além de ser realizada por um único avaliador. Cabe citar, que pelo tamanho da amostra, o método recordatório apresentou-se o mais adequado e os instrumentos utilizados são amplamente difundidos e utilizados pela literatura internacional.

O presente estudo verificou que os comportamentos relacionados à saúde e indicadores metabólicos em universitários, durante a exposição ao ambiente da graduação, sofreram alterações. $\mathrm{O}$ tempo de graduação explicou a prática de AFMV com uma variância de $16 \%$, o consumo de bebidas alcoólicas em 13,0\% e $14,0 \%$ (consumo em excesso), a medida da cintura em $13,0 \%$, e a pressão arterial em 9,0\% (PAS) e $6 \%$ (PAD). Os dados demonstram que cerca de $80,0 \%$ da variância dos comportamentos relacionados à saúde e indicadores metabólicos em universitários não pode ser explicado pelo tempo de exposição. Indicando que é necessário novas pesquisas que expliquem as variáveis que predizem as mudanças na prática de atividade física, comportamentos relacionados à saúde e indicadores metabólicos em universitários.

\section{Conflito de interesses}

Os autores declaram não haver conflito de interesses.

\section{Financiamento}

Pesquisa financiado pelos próprios autores.

\section{Contribuições dos autores}

Gasparotto GS, participou da concepção inicial do estudo, revisão da literatura, coleta de dados, análise dos dados e redação do artigo em todas as suas etapas. Pacífico $\mathrm{AB}$, participou da revisão da literatura e redação do artigo em todas as suas etapas. Camar- go EM, participou da revisão da literatura e redação do artigo em todas as suas etapas. Campos W, foi responsável pela revisão crítica do manuscrito. Todos os autores aprovaram a versão final do manuscrito.

\section{Referências}

1. Varela-Mato V, Cancela JM, Ayan C, Martín V, Molina A. Lifestyle and Health among Spanish University Students: Differences by Gender and Academic Discipline. Int J Environ Res Public Health. 2012;40(9):2728-41.

2. Plotnikoff RC, Costigan SA, Williams RL, Hutchesson MJ, Kennedy SG, Robards SL, et al. Effectiveness of interventions targeting physical activity, nutrition and healthy weight for university and college students: a systematic review and meta-analysis. Int J Behav Nutr Phys Act. 2015;12(45):1-10.

3. Ramis TR, Mielke GI, Habeyche EC, Oliz MM, Azevedo MR, Hallal PC. Tabagismo e consumo de álcool em estudantes universitários: prevalência e fatores associados. Rev Bras Epidemiol. 2012;15(2):376-85.

4. Gasparotto GS, Fantineli ER, Campos W De. Tobacco use and alcohol consumption associated with sociodemographic factors among college students. Acta Sci. 2015;37(1):11-18.

5. Mellado VC, Avaca CV, Gómez-Campos R, Rocha CL, Cossio-Bolaños M.Tendencias al incremento de la adiposidad corporal y la presión arterial de jóvenes universitarios en dos cohortes (2009-2014). Nutr Hosp. 2015;32(6):2551-58.

6. Rombaldi AJ, Silva MC, Dumith SC, Viana VR, Hallal PC. Fatores de risco para doenças crônicas não transmissíveis em estudantes de educação física ingressantes e formandos. Rev Bras Ciênc Esporte. 2014;36(1):13-27.

7. Franca C, Colares V. Estudo comparativo de condutas de saúde entre universitários no início e no final do curso. Rev Saúde Pública. 2008;42(3):420-27.

8. Gropper SS, Simmons KP, Connell LJ, Ulrich P V. Changes in body weight, composition, and shape: a 4-year study of college students. Appl Physiol Nutr Metab. 2012;37(6):1118-23.

9. Anstey KJ, Hofer SM. Longitudinal designs, methods and analysis in psychiatric research. Aust N Z J Psychiatry. 2004;38(1):93-104.

10. Gasparotto GS. Association between moment of the undergraduate course and cardiovascular risk factors in university students. Rev Latino-Am Enfermagem. 2013;21(3):687-94.

11. Pardini R, Matsudo S, Araújo T, Matsudo V, Andrade E, Braggion $\mathrm{G}$, et al. Validação do questionário internacional de nível de atividade física (IPAQ-versão 6): estudo piloto em adultos jovens brasileiros. Rev Bras Ciên e Mov. 2001;9(3):45-51.

12. Teixeira M. YRBS-C: Tradução, adaptação transcultural e propriedades psicométricas. Tese de Doutorado. [Londrina]: Universidade Estadual de Londrina - UEL; 2009.

13. Campos LL, Isensse DC, Rucker TC, Bottan ER. Condutas de saúde de universitários ingressantes e concluintes de cursos da área da saúde. Rev Bras Pesqui em Saúde. 2017;18(2):17-25.

14. Tarnus E, Bourdon E. Anthropometric evaluations of body composition of undergraduate students at the University of La Réunion. Adv Physiol Educ. 2006;30(4):248-53.

15. Sociedade Brasileira de Cardiologia. VI Diretrizes Brasileiras de Hipertensão. Arq Bras Cardiol 2010; 95(1 supl.1): 1-51. http://publicacoes.cardiol.br/2014/diretrizes.asp. 
16. Moreira CA, Lima FM, Silva PN. A difícil tarefa de acadêmicos de curso noturno em conciliar trabalho e estudo. Rev Eletronica da Univar. 2011;6(4):51-56.

17. Jacobsen N, Jensen H, Goldschmidt E. Does the Level of Physical Activity in University Students Influence Development and Progression of Myopia? A 2-Year Prospective Cohort Study. Assoc Res Vis Ophthalmol. 2008;49(4):1322-27.

18. Gasparotto G da S, Moreira NB, Gasparotto LPR, Silva MP, Campos. Associação entre fatores sociodemográficos e o nível de atividade física de universitários. Ciência e Mov. 2013;21(4):30-40.

19. Benotsch EG, Snipes DJ, Martin AM, Bull SS. Sexting, Substance Use, and Sexual Risk Behavior in Young Adults. J Adolesc Heal. 2013;52(3):307-13.

20. O'Grady KE, Arria AM, Fitzelle DMB, Wish ED. Heavy Drinking and Polydrug Use among College Students. J Drug Issues. 2008;38(2):445-65.

21. Iwamoto DK, Corbin W, Fromme K. Trajectory classes of heavy episodic drinking among Asian American college students. Addiction. 2010;105(11):1912-20.

22. Halperin AC, Smith SS, Heiligenstein E, Brown D, Fleming MF. Cigarette smoking and associated health risks among students at five universities. Nicotine Tob Res. 2010;12(2):96-104.

23. Lynn A. Body Mass Index Trends and Nutrition Goals of College Students between 2007-2011. Thesis. [Lincon]: University of Nebraska; 2012.

24. Lloyd-Richardson EE, Bailey S, FavaJL, Wing RA.Prospective Study of Weight Gain During the College Freshman and Sophomore Years. Prev Med. 2010; 48(3):256-61.
25. Goff DC Jr, Lloyd-Jones DM, Bennett G, et al.. 2013 ACC/ AHA guideline on the assessment of cardiovascular risk: a report of the American College of Cardiology/American Heart Association Task Force on Practice Guidelines. Circulation. 2014;129(25 Suppl 2):S49-73

26. Morrow ML, Heessch K, Dinger MK, Hull HR, Kneehans AW, Fields DA. Freshman 15: Fact or Fiction? Obesity. 2006;14(8):1438-43.

27. Vadeboncoeur C, Townsend N, Foster C. A meta-analysis of weight gain in first year university students: is freshman 15 a myth? BMC Obes. 2015; 2(1): 22-29.

28. Al-Majed HT, Sadek AA. Pre-hypertension and hypertension in college students in Kuwait: A neglected issue. J Fam Community Med. 2012;19(2):105-15.

29. Martins $M$ do $\mathrm{C}$ de $\mathrm{C}$ e, Ricarte IF, Rocha CHL, Maia RB, Silva VB da, Veras AB, et al. Blood pressure, excess weight and level of physical activity in students of a public university. Arq Bras Cardiol. 2010;95(2):192-99.

30. Sampson UKA, Edwards TL, Jahangir E, Munro $H$, Wariboko M, Wassef MG, et al. Factors Associated With the Prevalence of Hypertension in the Southeastern United States. Circ Cardiovasc Qual Outcomes. 2013; 7(1):33-54.

Recebido: 30/06/2017

Aprovado: 30/11/2017

\section{Como citar este artigo: \\ Gasparotto GS, Pacífico AB, Camargo EM, Campos W. Mudanças em comportamentos relacionados à saúde e indicadores metabólicos em universi- tários entre 2011 e 2014. Rev Bras Ativ Fís Saúde. 2017; 22(5):471-78. DOI: 10.12820/rbafs. v.22n5p471-78.}

